\title{
Efecto de la Relación de Compresión en el Rendimiento de Motores de Combustión Interna a diferentes Altitudes
}

\author{
José Miralles, Alberto Giménez*, Luis Domenech y Victor García \\ Escuela Superior de Enseñanzas Técnicas, Universidad CEU Cardenal Herrera, \\ C/ San Bartolomé 55. Alfara del Patriarca, Valencia 46115-España. \\ (e-mail: ppmiralles@telefonica.net; algisan@uch.ceu.es*; luis.domenech@uch.ceu.es; vicgarpe@uch.ceu.es
}

*Autor a quien debe ser dirigida la correspondencia

Recibido Dic. 22, 2014; Aceptado Mar. 4, 2015; Versión final Abr. 24, 2015, Publicado Ago. 2015

\begin{abstract}
Resumen
Se desarrollan ecuaciones analítico-empíricas para determinar la pérdida de potencia de motores de combustión interna alternativos (ciclo Otto) a distintas altitudes en función de la relación de compresión del motor y los coeficientes adiabáticos del aire-combustible y del aire puro. La disminución de la densidad del aire con la altitud es la causa más importante de la disminución de la potencia en un motor de combustión interna alternativo. Sin embargo, existen otros factores que producen $\mathrm{m}$ disminución de la potencia, y que los modelos actuales no consideran. Se obtiene una expresión que, además de considerar la densidad del aire, añade la relación de compresión del motor. Así la pérdida de potencia debido a la altitud, para un motor de combustión interna alternativo con una potencia determinada a nivel del mar, es ligeramente menor a mayor relación de compresión.
\end{abstract}

Palabras clave: motores de combustión interna, eficiencia de un motor, perdida potencia, rendimiento en altitud

\section{Effect of the Compression Ratio on the Efficiency of Internal Combustion Engines at different Altitudes}

\begin{abstract}
Considering these factors, this study developed analytical-empirical equations to obtain power losses in internal combustion engines (Otto cycle) at a different altitude based on the compression ratio of the engine and on the adiabatic coefficients of air-fuel air and pure air. The decrease in air density with altitude is the most important cause for the power loss of internal combustion engines. However, there are other factors that introduce additional power reduction, and that are not considered in current models available. An expression that not only considers the density of air but also includes the compression ratio of the engine is proposed. Thus, the power loss due to the altitude for an internal combustion engine with a certain power at sea level, is slightly lower at higher compression ratios.
\end{abstract}

Keywords: internal combustion engines, engine efficiency, power losses, efficiency in altitude 


\section{INTRODUCCIÓN}

El presente estudio surge como consecuencia de los trabajos de investigación realizados para mejorar las prestaciones en los motores de combustión interna alternativos de encendido provocado (MCIA-MEP), utilizados en plantas de potencia de aviones no tripulados (VANT ó UAV) de reducido tamaño y destinados a operar a media y gran altitud. Asimismo, uno de los requisitos que se les exige a estos aviones en esta investigación es el de gran autonomía (MALE y HALE) y cuyo proceso de investigación y desarrollo culmina con la fabricación de dos aviones no tripulados: El primero de ellos de categoría MALE (con peso al despegue inferior a $25 \mathrm{Kgf}$, techo máximo de servicio de $4000 \mathrm{~m}$, alcance máximo de $4000 \mathrm{Km}$ y autonomía de vuelo superior a 30 horas) utiliza como planta de potencia un minimotor de aspiración natural, monocilíndrico de cuatro tiempos y ciclo Otto, acoplado directamente a una hélice de alta eficiencia. El segundo avión, de categoría HALE (peso al despegue inferior a $12 \mathrm{Kg}$, techo máximo de servicio mayor de $9000 \mathrm{~m}$, alcance máximo de $2000 \mathrm{Km}$ y autonomía de vuelo mayor de 20 horas), utiliza una planta de potencia híbrida basada en un MCIA-MEP con características similares al motor citado anteriormente, un arrancador-generador eléctrico acoplado al mismo, un pack de baterías tampón y un motor eléctrico de alta eficiencia acoplado a una hélice. Para ambos aviones se efectuaron multitud de pruebas con motores de distintas cilindradas ( 25 c.c., 35 c.c. y 60 c.c.), adaptándolos a las condiciones operativas requeridas, permitiendo que los motores trabajen con distintos combustibles -gasolina, alcohol etílico, alcohol metílico y queroseno (previos ajustes)- y a diferente altitud.

Uno de los problemas que se nos presenta en el desarrollo de aviones de reducido tamaño (McCormick, Reymar, Torenbeck) y que se pretende resolver en el presente artículo es la pérdida de potencia en un motor de combustión interna alternativo (MCIA) de aspiración natural con la altitud, máxime teniendo en cuanta la escasa potencia disponible en estos pequeños motores.

La disminución de la densidad del aire con la altitud es la causa más importante de la disminución de la potencia, de esta forma ya en 1943 y tras el ensayo de distintos motores para aviación, Hislop estimó que no existe ninguna alteración en las pérdidas de potencia mecánicas conforme cambia la altitud, y concluyó que la variación de la potencia con la altitud en los motores de combustión interna alternativos (MCIA) de aspiración natural se puede expresar en función del ratio de densidad $\sigma$ y otros factores de corrección, de acuerdo con las ecuaciones empíricas siguientes:

$P_{\text {alt }}=P_{\text {sl }}(1.085 \sigma-0.085)$

$P_{\text {alt }}=P_{\text {sl }}(1.115 \sigma-0.115)$

Estas ecuaciones y otras similares se forjaron en los ensayos realizados por la sociedad londinense Aeronautical Research Council Reports and Memoranda, con el fin de evaluar la variación de potencia con la altitud (hasta $11000 \mathrm{~m}$ ) en los diferentes tipos de motores Merlin de ciclo Otto instalados en los aviones Spitfaire. Se analizaron multitud de variables, y las ecuaciones se ajustaron según las condiciones de trabajo de cada tipo de motor. Dichas ecuaciones fueron utilizadas como referencia para la estimación de las pérdidas de potencia en altitud. Siguiendo estos estudios, las normas MAPA-EMA (1960), SAE J1349 (1980), DIN 70020 (1986) y UNE 68028 (1986) definen ecuaciones que sirven para predecir las características de un MCIA conforme cambian la presión y la temperatura del aire troposférico, en un rango de baja y media altitud y en unas condiciones de trabajo especificadas para cada una de las citadas normas y que otros autores toman como referencia actualmente para la determinación de las pérdidas de potencia (Hesse, Mathews, Mattingly, Nickol, Palethorpe y Scholtz, entre otros).

Posteriormente, Xiaoping et al. (1996), observan una disminución de la potencia con la altitud, si bien argumentan que entre los factores de dicha disminución y de forma específica las pérdidas de potencia mecánica, por rozamiento y por bombeo se reducen levemente con la altitud. Finalmente indican que la variación de potencia con la altitud, debido sólo a las pérdidas de potencia mecánicas, es exigua frente a la potencia indicada y representa un porcentaje constante de la variación de la potencia indicada por efecto de la altitud. Agudelo et al. (2009), asumen que la reducción de la presión atmosférica con la altitud, aparte de otros fenómenos, provoca una menor presión final o menor presión de compresión en la cámara de combustión. Esto afecta las condiciones locales de combustión que modifican el ciclo termodinámico de operación del motor y, en consecuencia, disminuye el rendimiento indicado del mismo, lo que induce mayor consumo específico de combustible e incrementa la pérdida de potencia indicada. Es, a su vez, un factor de peso si consideramos potencias de trabajo pequeñas para el diseño de aviones que deben trabajar en altitud. Asimismo, Lapuerta et al. (2006), en la revista Información Tecnológica № 17, consideran que no existe alteración en las pérdidas de potencia mecánicas conforme cambia la altitud, por lo que sugieren idéntica alteración para la potencia indicada y para la efectiva, e igualmente para el rendimiento indicado y para el efectivo. 
Estudios más recientes llevados a cabo por Boehman A. y Pérez P. (2010) simulan condiciones de trabajo en altitud para el ajuste de motores monocilindricos utilizados en UAV, si bien el análisis se centra en motores diésel y en el enriquecimiento de oxígeno en la admisión en lugar de modificaciones en el diseño del mismo motor, como puede ser la relación de compresión. Boehman concluye que la potencia depende principalmente de la carga del motor y que esta no se mejora por el uso de aire enriquecido con oxígeno; por lo tanto, no se observan diferencias importantes en la pérdida de potencia hasta los 2600 metros de altitud. Actualmente, como modelo general para el cálculo de la potencia con la altitud de los MCIA de aspiración natural, especialmente para los destinados a la aviación, se utiliza una ecuación empírica derivada de las descritas arriba (1) y (2), con resultados igualmente válidos hasta los 11.000 metros de altitud, Gudmundsson (2013); Tulapurkara, E. G. (2008). Según Gudmundsson esta ecuación fue modelizada por Gagg y Ferrrar y se escribe como sigue:

$\mathrm{P}_{\mathrm{alt}}=\mathrm{P}_{\mathrm{sl}}\left(\sigma-\frac{1-\sigma}{7.55}\right)=\mathrm{P}_{\mathrm{sl}}\left(\frac{8.55 \sigma-1}{7.55}\right)=\mathrm{P}_{\mathrm{sl}}(1.13 \sigma-0.13)$

En esta ecuación, $P_{\text {alt }}$ es la potencia a la altitud considerada: $P_{\text {sl }}$ es la potencia indicada a nivel del mar, y $\sigma$ es la razón de densidad o coeficiente de densidad. $(\sigma \leq 1)$.

En la figura 1 se muestra el factor de potencia de un MCIA-MEP en función de la altitud $z$ según modelo de Gagg y Ferrrar (ecuación 3). En la figura se puede observar la divergencia del citado modelo con respecto de la razón de densidad $(\sigma)$ solo.

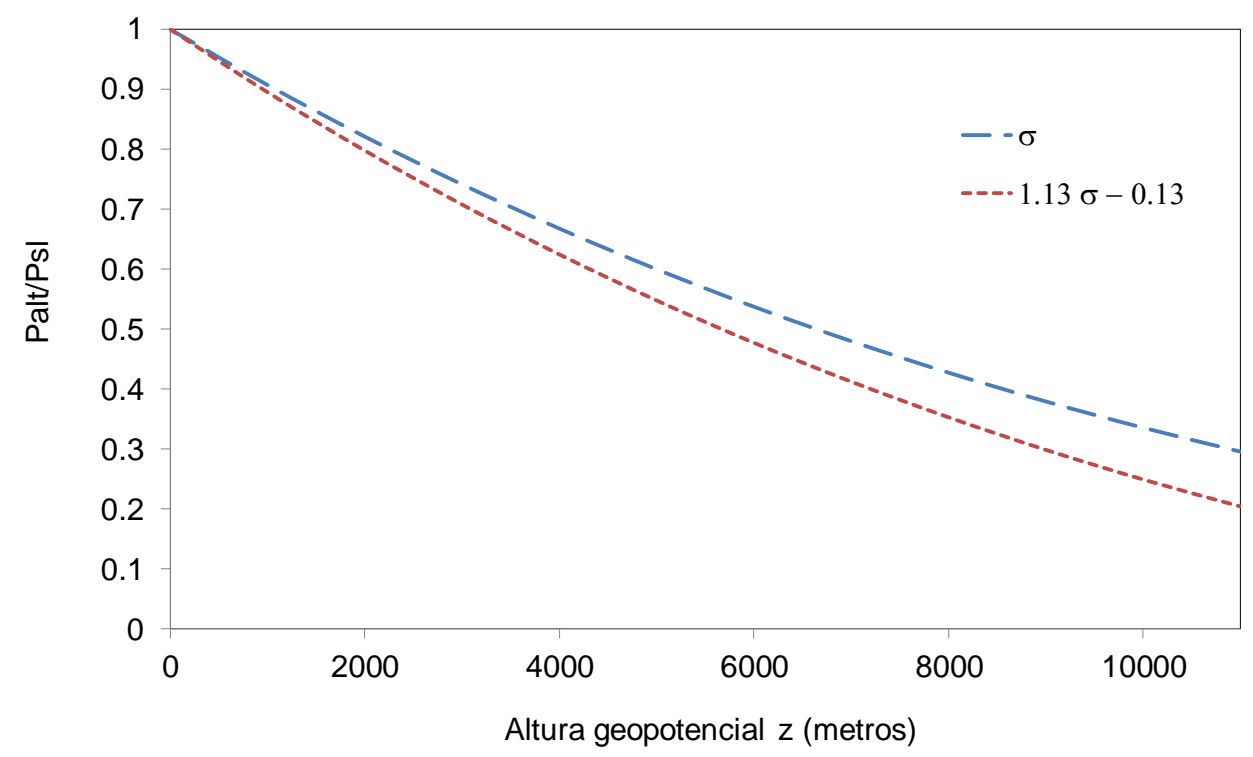

Fig. 1: Factor de potencia en función de la altitud $z$ (ISA) MCIA de ciclo Otto y de aspiración natural

En este punto y una vez indicadas las referencias encontradas al estudio de la pérdida de potencia de motores en altitud, se debe remarcar que tanto la ecuación modelizada por Gagg y Ferrar como las anteriores (1) y (2), o cualquier otra ecuación en uso no toman en consideración la relación de compresión del MCIA-MEP en estudio.

Atendiendo a la relación de compresión como un parámetro que afecta al factor de pérdidas de potencia en el estudio de los motores de los aviones en altitud y teniendo en cuenta las referencias bibliográficas (Austin, Glassock, Lee, Lieh, Merical y Meyer), se partió de una relación de compresión de 8:1. El desempeño de los mismos a nivel del mar o a baja altitud fue muy satisfactorio. Por el contrario, a media y gran altitud su desempeño era muy inestable, incluso con ajustes óptimos de dosado e ignición se producían paradas repentinas por encima de los $4000 \mathrm{~m}$ de altitud y, en mayor medida, con cargas parciales bajas. Asimismo, se efectuaron diversos ensayos en altitud con los citados motores utilizando gasolina de 95 y 98 octanos, constatándose que no se producían paradas repentinas cuando se aumentaba la relación compresión de estos motores desde 10.5:1 hasta 12:1, incluso a 14:1 para gran altitud, manteniendo la carga por encima del $50 \%$.

En los referidos experimentos, se pudo evidenciar que conforme se aumentaba la relación de compresión, la pérdida de potencia en función de la altitud, respecto de la potencia a nivel del mar, era ligeramente menor (acorde a una ley no lineal) que la proporcionada por las ecuaciones indicadas. Por ello se consideró 
que debía añadirse un factor adicional para la determinación de la pérdida de potencia que contemplara la relación de compresión de los motores.

Para mejor aclaración, sirva el ejemplo siguiente. Supongamos dos MCIA-MEP iguales con relaciones de compresión 7:1 y 13:1. Sus potencias serán lógicamente diferentes, siendo mayor la del motor con mayor relación de compresión. Con las ecuaciones en uso para el cálculo de la potencia de los MCIA-MEP en altitud (estas ecuaciones no consideran la relación de compresión), el factor que multiplica a la potencia a nivel del mar sería igual para ambos motores. Sin embargo, según nuestra experiencia, este factor se incrementa con la altitud, conforme aumenta la relación de compresión. Esto implica una menor pérdida de potencia para el motor con relación de compresión 13:1, y una mayor pérdida de potencia para el motor con relación de compresión 7:1. Se efectuaron numerosas pruebas con parejas de motores idénticos, pero con distintas relaciones de compresión, lo que ha permitido establecer un modelo matemático para el cálculo de la pérdida de potencia de los MCIA-MEP en función de la altitud, y para lo cual se expone el presente modelo.

\section{DESARROLLO DEL MODELO}

\section{Merma de la potencia con la altitud en los MCIA}

La potencia de un motor de combustión interna alternativo (MCIA) es directamente proporcional a la masa de aire que se consigue introducir en el cilindro, por unidad de tiempo o por embolada (Agüera, Morán, Sala). Dicha masa de aire es función del volumen del cilindro y de la densidad del aire. Si admitimos un motor ideal de cuatro tiempos con un cilindro de de volumen $V$, sin restricciones a la entrada de admisión y trabajando atmósfera ISA, la masa de este aire que ingresará en una embolada será:

$m=\rho V$

La densidad del aire disminuye con la altitud, por lo que la potencia indicada disminuirá conforme se reduzca esta densidad, de acuerdo a la proporción:

$\sigma=\frac{\rho_{d}}{\rho_{\mathrm{o}}}$

En esta ecuación, $\sigma$ es la razón de densidades o coeficiente de densidades $(\sigma \leq 1)$, $\rho_{d}$ es la densidad del aire seco según la altitud (atmósfera ISA) y po es la densidad estándar a nivel del mar (Atmósfera ISA: 1.225 $\mathrm{Kg} / \mathrm{m}^{3}$ ). Si en la expresión (5), sustituimos el valor de $\rho$ d por la expresión que sigue (corolario de las ecuaciones que definen el aire seco en la troposfera en condiciones estándar ISA (Ledesma, Minzner, Barry), entonces tendremos que:

$\sigma=\frac{\rho_{d}}{\rho_{0}}=\left[1-\frac{\lambda z}{T_{0}}\right]^{\left(g / R^{\prime} d \lambda\right)-1}=\left[1-\frac{6.5 \cdot 10^{-8} z}{288.15}\right]^{4.2559}$

Ecuación que proporciona la representación gráfica y que, en una atmósfera ISA, nos indica el ratio o coeficiente de densidad en función de la altura geopotencial $z$ de un punto ubicado en la troposfera. La figura muestra la razón de densidad en función de la altitud $z$ en metros (atmósfera ISA).

Para un motor de combustión interna alternativo (MCIA) de aspiración natural y encendido provocado (MEP), trabajando a una altitud establecida en la troposfera, con unos ajustes óptimos de dosado e ignición, y conforme a lo expuesto en este apartado, cabría pensar que la potencia desarrollada por este motor, respecto a la del nivel del mar se podría deducir en función de la densidad del aire (Pairy, Muñoz) de acuerdo con la expresión siguiente:

$P_{\text {alt }}=\sigma P_{\text {sl }}$

Palt, Potencia a la altitude considerada; $P_{s l}$, Potencia indicada a nivel del mar; $\sigma$, Razón de densidad 0 coeficiente de densidad.

Ciertamente se puede demostrar que la anterior ecuación no resulta suficiente para predecir la potencia de un MCIA-MEP a determinada altitud con respecto a la del nivel del mar, ya que arroja pérdidas de potencia inferiores a la realidad, con valores inexactos que aumentan conforme se acrecienta la altitud, por lo que será necesario aplicar un segundo factor de corrección. 


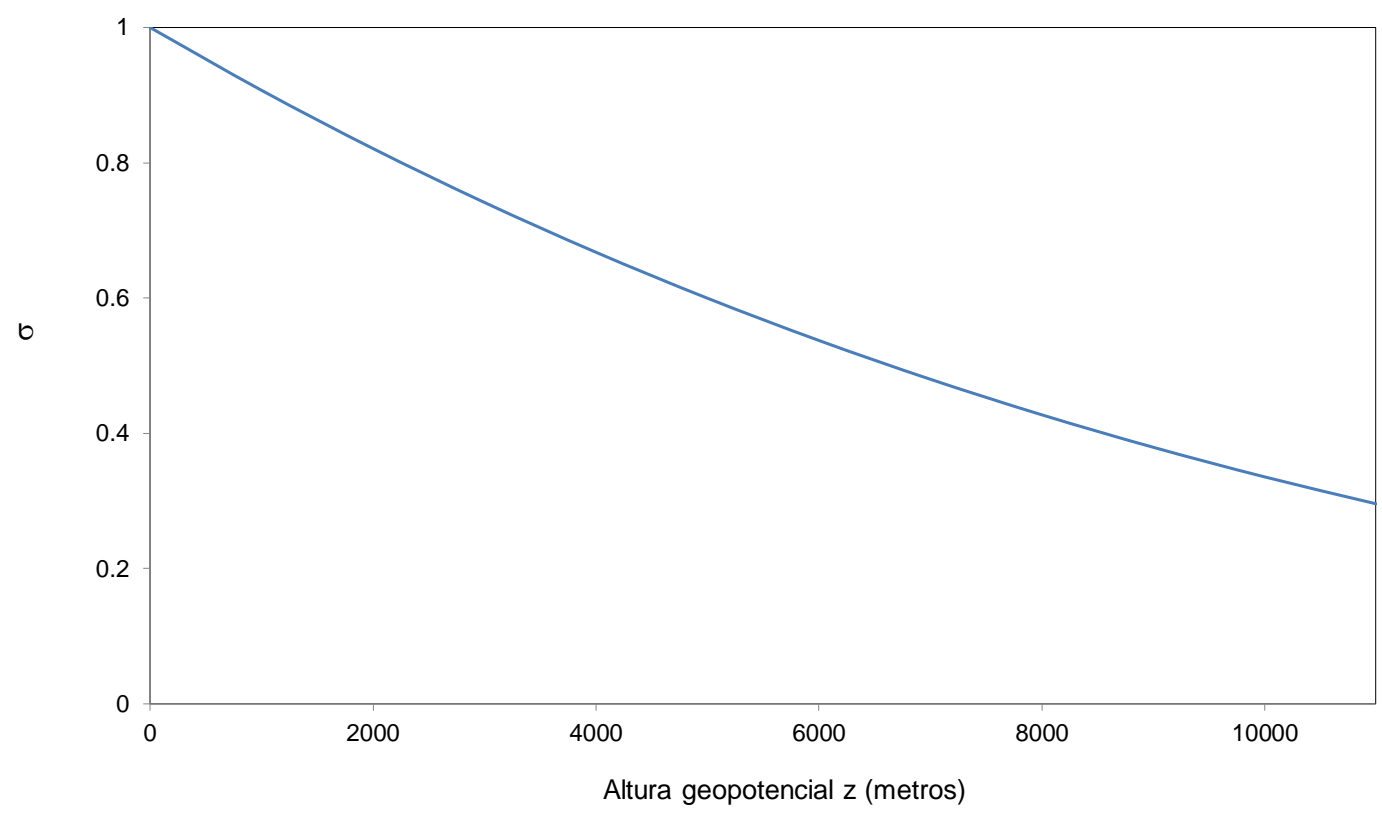

Fig. 2: Razón de densidad en función de la altitud $z$ en metros (atmósfera ISA)

\section{Coeficiente de incremento de pérdida de potencia según la altitud}

De acuerdo con lo expresado anteriormente, la reducción de la presión atmosférica debido a la altitud afecta a las condiciones locales de combustión en un MCIA de aspiración natural. Ello introduce modificaciones en el ciclo termodinámico de operación del motor y, en consecuencia, se menoscaba el rendimiento indicado del mismo.

De este modo, la potencia del motor a determinada altitud se podrá escribir mediante un término que designaremos como factor de potencia $(\sigma \cdot \delta)$, el cual tiene en cuenta el ratio de densidad $(\sigma)$ y otro coeficiente que denominaremos, "coeficiente de incremento de pérdida de potencia según la altitud" $(\delta)$, conforme a la expresión siguiente:

$\mathrm{P}_{\mathrm{alt}}=\sigma \cdot \delta \cdot \mathrm{P}_{\mathrm{sl}}$

En esta ecuación, $P_{\text {alt }}$ es la potencia a la altitud considerada, $P_{s l}$ es la potencia indicada a nivel del mar $\sigma$ Razón de densidad o coeficiente de densidad, y $\delta$ es el coeficiente de incremento de pérdida de potencia según altitud $(\delta \leq 1)$

Podemos teorizar que el coeficiente de incremento de pérdida de potencia $\delta$ es función de las modificaciones que sufre el ciclo termodinámico del MCIA por efecto de la altitud, lo que a su vez altera el rendimiento indicado. Así mismo, se ha probado empíricamente que el valor de $\delta$ para un MCIA de ciclo Otto queda afectado por su relación de compresión y por la razón de presiones entre el nivel del mar y la altitud considerada, conforme a la expresión siguiente:

$\delta=\left[\frac{r^{\gamma-1}-\left[\frac{p_{0}}{p_{d}}\right]^{(\gamma-1) / \gamma}}{r^{\gamma-1}-1}\right]^{n}$

En esta ecuación las variables significan: pd, Presión atmosférica del aire seco a la altitud considerada; po, Presión atmosférica estándar a nivel del mar $(1013,25 \mathrm{hPa}) ; r$, Relación volumétrica 0 relación de compresión; $\gamma$, Coeficiente adiabático aire gasolina $(\gamma=1,35)$; $n$, Coeficiente politrópico de ajuste $(n=0,5)$

La ecuación (8) se puede escribir ahora como sigue: 
$\frac{P_{\text {alt }}}{P_{\mathrm{sl}}}=\sigma \cdot \delta=\frac{\rho_{d}}{\rho_{\mathrm{o}}}\left[\frac{r^{\gamma-1}-\left[\frac{p_{\mathrm{o}}}{\mathrm{p}_{\mathrm{d}}}\right]^{(\mathrm{\gamma}-1) / \gamma}}{\mathrm{r}^{\gamma-1}-1}\right]^{1 / 2} \rightarrow \frac{\mathrm{P}_{\text {alt }}}{\mathrm{P}_{\mathrm{sl}}}=\sigma \cdot \delta=\frac{\rho_{\mathrm{d}}}{\rho_{\mathrm{o}}}\left[\frac{\mathrm{r}^{0.35}-\left[\frac{\mathrm{p}_{\mathrm{o}}}{\mathrm{p}_{\mathrm{d}}}\right]^{\frac{0.35}{1.35}}}{\mathrm{r}^{0.35}-1}\right]^{0.5}$

Cabe reseñar que el modelo expuesto para determinar el factor de potencia $(\sigma \cdot \delta)$ en función de la altitud, para un motor de combustión interna alternativo de encendido provocado (MCIA-MEP), no difiere de los estándares en uso en cuanto a las variables implicadas, es decir, presión y densidad atmosféricas, además de la temperatura implícita en ambas variables. En este caso se añade la variable relación volumétrica o relación de compresión (r).

Por otra parte, según la Atmósfera Estándar Internacional ISA, las ecuaciones que definen el aire seco en la troposfera (Barry, Tsonis, Wallace), desde el nivel del mar hasta los once mil metros (11 $000 \mathrm{~m})$, son las siguientes:

$\mathrm{T}_{\mathrm{z}}=\mathrm{T}_{\mathrm{o}}-\lambda \cdot \mathrm{z}$

$\rho_{d}=\rho_{o}\left[\frac{T_{0}-\lambda z}{T_{0}}\right]^{\left(g / R_{d}^{\prime} \lambda\right)-1}=\rho_{o}\left[1-\frac{\lambda z}{T_{0}}\right]^{\left(g / R{ }_{d}^{\prime} \lambda\right)-1}$

$p_{d}=p_{o}\left[\frac{T_{0}-\lambda z}{T_{0}}\right]^{\left(g / R{ }^{\prime} \lambda\right)}=p_{o}\left[1-\frac{\lambda z}{T_{0}}\right]^{\left(g / R{ }^{\prime} \lambda\right)}$

En estas ecuaciones los símbolos significan lo que sigue:

$\mathrm{T}_{\mathrm{z}}$, Temperatura de altitud en $\mathrm{K}$

$\mathrm{T}_{\mathrm{o}}$, Temperatura estándar a nivel del mar $(273+15 \mathrm{~K})$

$\lambda$, Gradiente térmico negativo en valor absoluto $\left(6,5 \cdot 10^{-3} \mathrm{~K} / \mathrm{m}\right)$

$z$, Altura geopotencial en metros

$\mathrm{p}_{\mathrm{d}}$, Presión atmosférica del aire seco a la altitud considerada

po, Presión atmosférica estándar a nivel del mar $(1013,25 \mathrm{hPa})$

$\rho d$, Densidad del aire seco según la altitud (atmósfera ISA)

$\rho_{0}$, Densidad estándar a nivel del mar (Atmósfera ISA: $1.225 \mathrm{Kg} / \mathrm{m}^{3}$ )

$g$, Valor promedio de la aceleración gravitacional $\left(9,8065 \mathrm{~m} / \mathrm{s}^{2}\right)$

$\mathrm{R}_{\mathrm{d}}$, Constante másica del aire seco $(287,05 \mathrm{~J} / \mathrm{Kg} \mathrm{K})$

$\mathrm{R}$, Constante de los gases perfectos $(8,3143 \mathrm{~J} / \mathrm{mol} \mathrm{K})$

$M_{d}$, Masa molar del aire seco $(0,0289644 \mathrm{Kg} / \mathrm{mol})$

De las expresiones anteriores se puede deducir que:

$\rho_{d}=\rho_{o}\left[\frac{T_{0}-\lambda z}{T_{0}}\right]^{\left(g / R^{\prime}{ }^{\prime} \lambda\right)-1} \rightarrow \rho_{d}=\left[\frac{44330.769-z}{42266.482}\right]^{4.2559} \frac{\mathrm{Kg}}{\mathrm{m}^{3}}$

$p_{d}=p_{o}\left[\frac{T_{0}-\lambda z}{T_{0}}\right]^{\left(g / R_{d}^{\prime} \lambda\right)} \rightarrow p_{d}=\left[\frac{44330.769-z}{4946.588}\right]^{5.2559} \mathrm{~Pa}$

Por lo que los cocientes de densidad y de presión de la ecuación (10) se pueden escribir como sigue:

$\frac{\rho_{d}}{\rho_{0}}=\left[\frac{T_{0}-\lambda z}{T_{o}}\right]^{\left(g / R_{d}^{\prime} \lambda\right)-1}=\left[1-225577 \cdot 10^{-10} z\right]^{4.2559}=\left[1-\frac{z}{44330.769}\right]^{4.2559}$

$\frac{p_{o}}{p_{d}}=\left[\frac{T_{0}-\lambda z}{T_{o}}\right]^{\left(-g / R_{d}^{\prime} \lambda\right)}=\left[1-225577 \cdot 10^{-10} z\right]^{-5.2559}=\left[1-\frac{z}{44330.769}\right]^{-5.2559}$

\section{ANÁLISIS DE RESULTADOS}

Con la aplicación de los datos de las ecuaciones (16) y (17) en la ecuación (10), se puede determinar y grafiar el factor de potencia $(\sigma \cdot \delta)$ de un MCIA de ciclo Otto de aspiración natural, con una relación de 
compresión definida, hasta una altitud de 11000 metros, como se observa en la figura 3. Así pues, aparte de las razones de densidad y de presión, el valor del factor de potencia en altitud $(\sigma \cdot \delta)$ evolucionará en función de la relación de compresión, creciendo o decreciendo ligeramente acorde a una mayor o menor magnitud del mismo. Sustituyendo en la ecuación (10) el valor de r, por la relación de compresión típica (7.2:1) para los MCIA de aviación de ciclo Otto, entonces se tiene:

$\frac{P_{\text {alt }}}{P_{\mathrm{sl}}}=\sigma \cdot \delta=\frac{\rho_{d}}{\rho_{\mathrm{o}}}\left[2-\left[\frac{p_{\mathrm{o}}}{p_{\mathrm{d}}}\right]^{\frac{0.35}{1.35}}\right]^{0.5}$

Si en la ecuación (18) expresamos el factor de potencia $(\sigma \cdot \delta)$ en función de la altura geopotencial $z$ (atmósfera ISA), tendremos:

$\frac{\mathrm{P}_{\mathrm{alt}}}{\mathrm{P}_{\mathrm{sl}}}=\sigma \cdot \delta=\left[1-\frac{\mathrm{z}}{44330.769}\right]^{4.22559}\left[2-\left[1-\frac{\mathrm{z}}{44330.769}\right]^{(-1.362)}\right]^{0.5}$

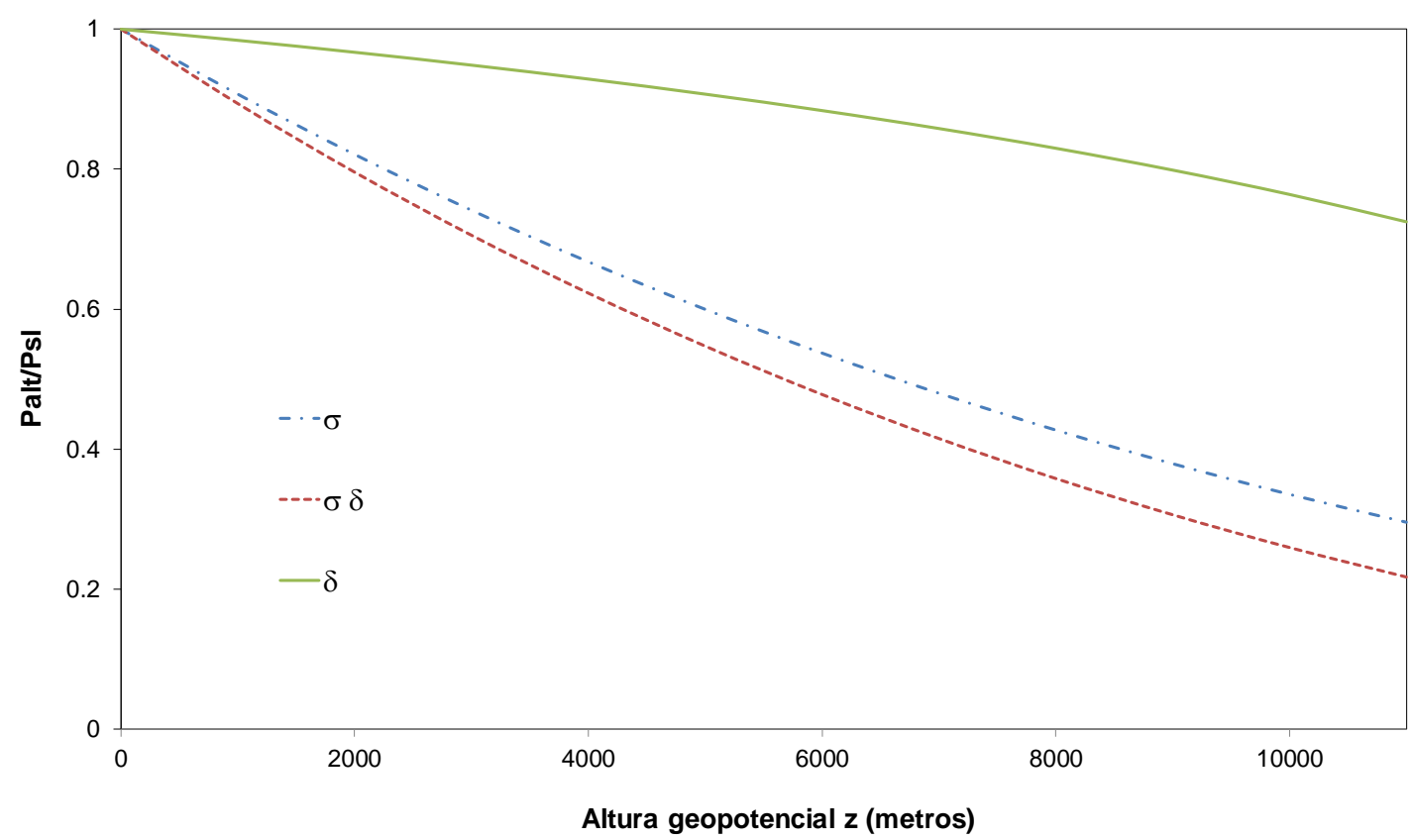

Fig. 3: Factor de potencia en función de la altitud en metros (atmósfera ISA) para un motor de ciclo Otto con $r=7.2: 1$.

En la figura 4 se compara la ecuación (3) del modelo de Gagg y Ferrrar con la ecuación aquí teorizada (10). Se asume que se trata de un motor de combustión interna alternativo de encendido provocado (MCIA-MEP). Para un coeficiente adiabático de la mezcla aire-combustible de 1.35 y una relación de compresión 7.2:1, normal en los motores de aeronaves equipadas con MCIA-MEP, se observa que ambas ecuaciones son prácticamente coincidentes hasta los $10000 \mathrm{~m}$. La concomitancia entre las referidas ecuaciones representa un aval al modelo aquí descrito para determinar el valor del factor de potencia $(\sigma \cdot \delta)$ en altitud de un MCIAMEP para diferentes relaciones de compresión $(\sigma \cdot \delta \cong 1,13 \cdot \sigma-0,13)$

De acuerdo con la figura 5, la pérdida de potencia debido a la altitud, para un MCIA-MEP con una potencia determinada a nivel del mar, será ligeramente menor cuanto mayor sea la relación de compresión (ley no lineal). 


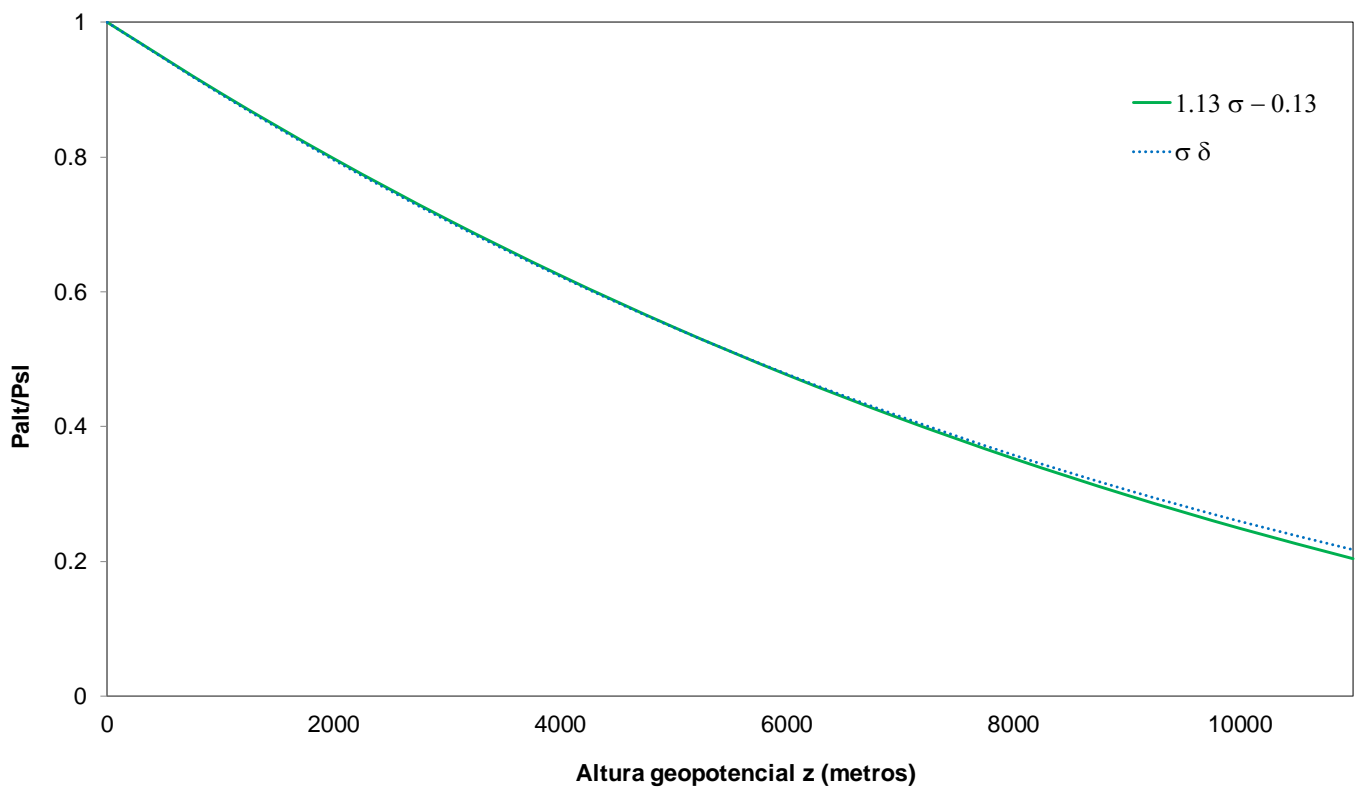

Fig. 4: Comparación del modelo de Gagg y Ferrrar y la ecuación propia (10) para un MCIA de ciclo Otto con relación de compresión típica para aviación $r=7.2: 1$

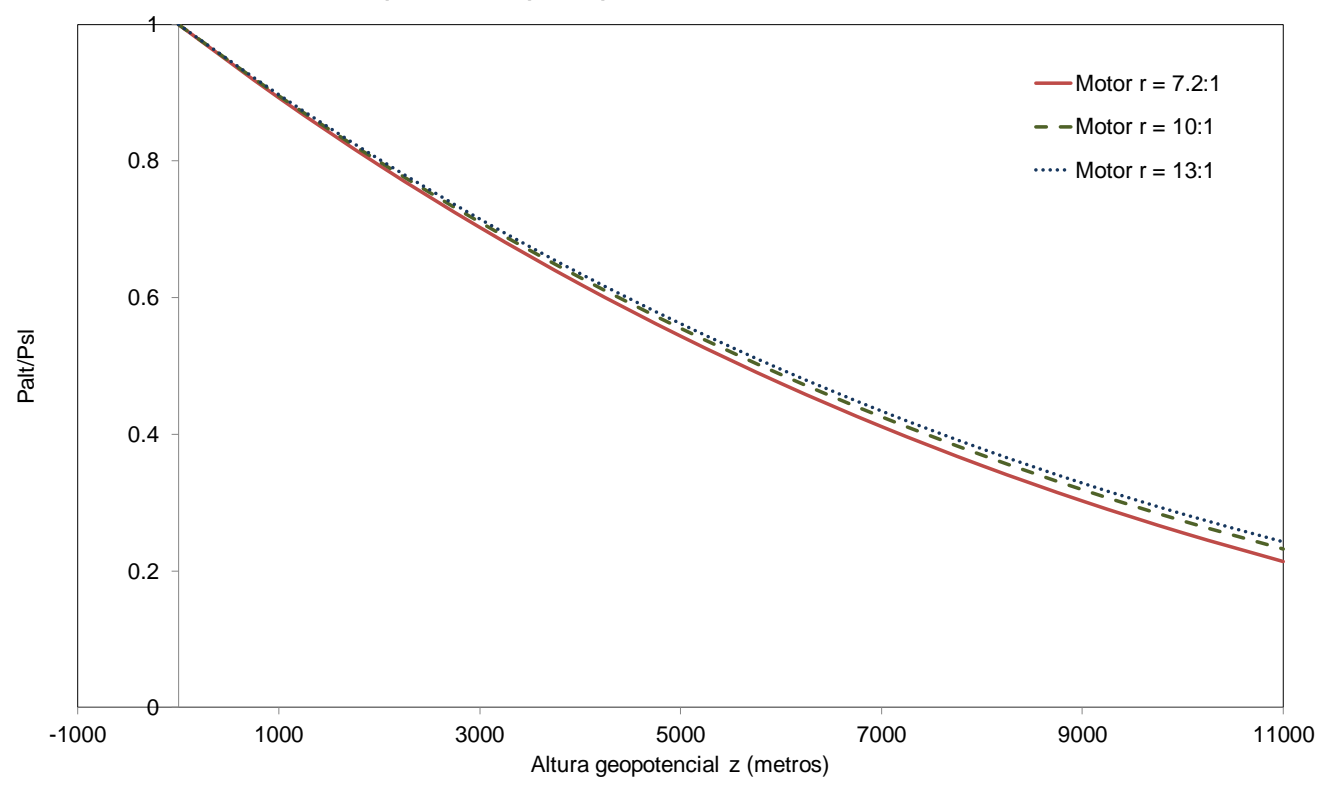

Fig. 5: Evolución estimada del factor de potencia en función de la altitud $z$ en metros. Modelo propio conforme a la ecuación (10) para MCIA-MEP, con relaciones de compresión de: 13:1, 10:1 y 7.2:1.

Por otra parte, si en la ecuación (10) sustituimos la densidad del aire seco $\rho_{d}$ por la densidad del aire húmedo $\rho_{a}$ deducida en condiciones atmosféricas verdaderas, acorde a la ecuación (20) de origen propio:

$\rho_{\mathrm{a}}=\frac{3.4837 \mathrm{p}-8.0489 \mathrm{HR} \mathrm{e}^{\frac{17.67 t}{\mathrm{t}+243.5}}}{10(273.15+\mathrm{t})}$

Siguiendo con la misma ecuación (10), si reemplazamos la presión pd por la presión total del aire húmedo $p$ deducida en condiciones atmosféricas verídicas mediante un sensor barométrico calibrado en $\mathrm{hPa}$, t en ${ }^{\circ} \mathrm{C}$ y HR en tanto por uno, entonces, el factor de potencia para una altitud imaginada en atmosfera verdadera, respecto a la estándar a nivel del mar, se podría expresar como:

$\frac{\mathrm{P}_{\text {alt }}}{\mathrm{P}_{\mathrm{sl}}}=\sigma \cdot \delta=\frac{\rho_{\mathrm{a}}}{1.225}\left[\frac{\mathrm{r}^{0.35}-\left[\frac{1013.25}{\mathrm{p}}\right]^{\frac{0.35}{1.35}}}{\mathrm{r}^{0.35}-1}\right]^{0.5}=\frac{3.4837 \mathrm{p}-8.0489 \mathrm{HR} \mathrm{e}^{\frac{17.67 t}{\mathrm{t}+243.5}}}{10(273.15+\mathrm{t})} \frac{1}{1.225}\left[\frac{\mathrm{r}^{0.35}-\left[\frac{1013.25}{\mathrm{p}}\right]^{\frac{0.35}{1.35}}}{\mathrm{r}^{0.35}-1}\right]^{0.5}$ 
Conforme a lo expresado en el párrafo anterior, sustituyendo la densidad $\rho_{a}$ y la presión $p$ efectivas en la ecuación (18), tendremos:

$\sigma \cdot \delta=\frac{3.4837 p-8.0489 \mathrm{HR} \mathrm{e}^{\frac{17.67 t}{t+243.5}}}{10(273.15+\mathrm{t}) 1.225}\left[2-\left[\frac{1013.25}{\mathrm{p}}\right]^{\frac{0.35}{1.35}}\right]^{0.5}$

Ecuaciones (21) y (22) que, en una atmósfera no ISA, nos indican el factor de potencia para una altitud determinada de un MCIA de ciclo Otto, para cualquier relación de compresión (r) y para una relación de compresión convencional $r=7.2: 1$ respectivamente, situado en un punto cualquiera de la troposfera y en función de las variables atmosféricas verdaderas calculadas en ese punto (densidad en $\mathrm{kg} / \mathrm{m}^{3}$, humedad relativa en tanto por uno, presión en $\mathrm{hPa}$ y temperatura en $\left.{ }^{\circ} \mathrm{C}\right)$.

\section{CONCLUSIONES}

En el presente artículo se han presentado una serie de ecuaciones que permiten determinar, la pérdida de potencia con la altura para motores de ciclo Otto y que suponen un avance con respecto a las ecuaciones utilizadas en la actualidad ( $y$ basadas en ensayos empíricos), lo que nos permite conocer mejor el comportamiento de un motor determinado ya que se han añadido factores adicionales no contemplados hasta la fecha.

La expresión aquí descrita para determinar la pérdida de potencia por efecto de la altitud en los motores de combustión interna alternativos de encendido provocado (MCIA-MEP) y aspiración natural, no difiere de los estándares en uso en cuanto a las variables implicadas, es decir, presión y densidad atmosféricas, además de la temperatura implícita en ambas variables.

Puesto que la densidad del aire seco es función de la presión y la temperatura atmosféricas, se puede decir que las variables realmente implicadas son estas dos últimas, además de la presión parcial de vapor de agua en atmósfera no estándar.

Se ha obtenido una expresión que, además de las variables indicadas, añade la relación volumétrica o relación de compresión del motor. Este modelo indica que la pérdida de potencia debido a la altitud, para un MCIA-MEP con una potencia determinada a nivel del mar, es ligeramente menor cuanto mayor es la relación de compresión. El modelo se ha ensayado con pequeños motores destinados a UAV hasta altitudes de $6000 \mathrm{~m}$, arrojando resultados satisfactorios.

En caso de considerar la atmósfera no estándar se pueden utilizar las ecuaciones derivadas de la ecuación (10) y deducidas en el presente artículo, es decir las ecuaciones (21) y (22).

\section{NOTACIÓN}

Palt, Potencia a la altitude considerada

$\mathrm{P}_{\mathrm{sl}}$, Potencia indicada a nivel del mar

$\sigma$, Razón de densidad o coeficiente de densidad. $(\sigma \leq 1)$

$\rho_{d}$, Densidad del aire seco según la altitud (atmósfera ISA)

$\rho_{0}$, Densidad estándar a nivel del mar (Atmósfera ISA: $1.225 \mathrm{Kg} / \mathrm{m}^{3}$ )

$\delta$, Coeficiente de incremento de pérdida de potencia según altitud $(\delta \leq 1)$

$r$, Relación volumétrica o relación de compresión

$\gamma$, Coeficiente adiabático aire gasolina $(\gamma=1,35)$

$\mathrm{n}$, Coeficiente politrópico de ajuste $(\mathrm{n}=0,5)$

$\mathrm{T}_{\mathrm{z}}$, Temperatura de altitud en $\mathrm{K}$

To, Temperatura estándar a nivel del mar $(273+15 \mathrm{~K})$

$\lambda$, Gradiente térmico negativo en valor absoluto $\left(6,5 \cdot 10^{-3} \mathrm{~K} / \mathrm{m}\right)$

z, Altura geopotencial en metros 
pd, Presión atmosférica del aire seco a la altitud considerada

po, Presión atmosférica estándar a nivel del mar (1013,25 hPa)

$g$, Valor promedio de la aceleración gravitacional $\left(9,8065 \mathrm{~m} / \mathrm{s}^{2}\right)$

Rd', Constante másica del aire seco $(287,05 \mathrm{~J} / \mathrm{Kg} \mathrm{K})$

$\mathrm{R}$, Constante de los gases perfectos $(8,3143 \mathrm{~J} / \mathrm{mol} \mathrm{K})$

$\mathrm{M}_{\mathrm{d}}$, Masa molar del aire seco $(0,0289644 \mathrm{Kg} / \mathrm{mol})$

\section{REFERENCIAS}

Agudelo, J.; Agudelo, A. y Pérez, J., Análisis energético y exergético de un motor diesel de automoción operando en diferentes altitudes. Revista Facultad de Ingeniería, Universidad de Antioquia. Medellín, Colombia, (2009)

Agüera, A., Termodinámica Lógica y Motores Térmicos. Ciencia 3 SL. Madrid, (1999)

Austin, R., Unmanned Aircraft Systems UAVS Design Development and Deployment, $1^{\text {a }}$ edición, Wiley, Chichester, United Kingdom (2010)

Barry, R.; Chorley, R., Atmósfera, tiempo y clima. $7^{a}$ edición, Omega, Barcelona, España (1999)

Boehman, A. y Pérez P. Performance of a single-cylinder diesel engine using oxygen-enriched intake air at simulated high-altitude conditions. Aerospace Science and Technology 14 (2), $83-94$ (2010)

Glassock, R.R.; Hung, J.Y.; Gonzalez, L.F. y Walker, R.A., Multimodal hybrid powerplant for unmanned aerial systems (UAS) robotics. Twenty-Fourth Bristol International Unmanned Air Vehicle Systems Conference, Bristol, United Kingdom 30 marzo al 1 abril (2009)

Gudmundsson, S., General Aviation Aircraft Design: Applied Methods and Procedures. $1^{\underline{a}}$ edición, Butterworth-Heinemann, Oxford, United Kingdom, (2014)

Hesse, W.J. y Mumford, N.V.S., Jet Propulsion for Aerospace Applications. $2^{a}$ edición, Pitman Publishing Corporation, New York, Estados Unidos(1995)

Hislop, G.S., The Variation of Power with Height of a Merlin 46 Engine as determined by Flight Tests on a Spitfire Vc. Aeronautical Research Council Reports and Memoranda, 2213, London (1943).

ISO/DIS 2533-1975, International Organization for Standardization, International Standard Atmosphere (ISA), Suiza (1972)

Lapuerta, M.; Armas, O.; Agudelo, J. y Sánchez, C.A. Estudio del Efecto de la Altitud sobre el Comportamiento de Motores de Combustión Interna. Parte 1: Funcionamiento. CIT Revista Información Tecnológica, 17 (5), 21-30 (2006)

Lapuerta, M.; Armas, O.; Agudelo, J. y Agudelo, A.F. Estudio del Efecto de la Altitud sobre el Comportamiento de Motores de Combustión Interna. Parte 2: Funcionamiento. CIT Revista Información Tecnológica, 17 (5), 31-41 (2006)

Ledesma, M., Principios de meteorología y climatología. 1a edición, Paraninfo, Madrid, España (2011)

Lee, B.; Park, P.; Kim, C.; Yang, S.; Ahn, S. Power managements of a hybrid electric propulsion system for UAVs. Journal of Mechanical Science and Technology, 26, 2291-2299 (2012)

Lieh, J.; Spahr, E.; Behbahani, A. y Hoying, J. Design of Hybrid Propulsion Systems for Unmanned Aerial Vehicles. Joint Propulsion Conference \& Exhibit, San Diego, Estados Unidos 31 julio al 3 agosto (2011)

Matthews, C., Aeronautical Engineer's Data Book. 1를 edición, Butterworth-Heineman, Oxford, UK (2002)

Mattingly, J.D.; Heiser, W.H. y Dailey, G.H. Aircraft Engine Design. 1a edición, American Institute of Astronautics and Aeronautics, Washington, United States (1987)

McCormick, B.W. Aerodynamics; Aeronautics; and Flight Dynamics. 2ª edición, Wiley, Nueva York, Estados Unidos (1995) 
Merical, K.; Beechner, T. y Yelvington, P. Hybrid-Electric Heavy-Fuel Propulsion System for Small Unmanned Aircraft. SAE International Journal of Aerospace, 7 (1), 126-134 (2014)

Meyer, J.; Plessis, F. y Clarke, W. Design Considerations for Long Endurance Unmanned Aerial Vehicles, doi: 10.5772/6482. Intech, 22, 443-498 (2009)

Minzner, R.A. The 1976 Standard Atmosphere and its relationship to earlier standards. Reviews of Geophysics, 15 (3), 375-384 (1977)

Morán, F. Apuntes de termodinámica de la atmósfera. Servicio Publicaciones del Instituto Nacional de Meteorología, Madrid, España (1984)

Muñoz, M.; Moreno, F. y Morea, J.F. Motores alternativos de combustión interna. 2ª edición, Editorial Universidad de Zaragoza, Zaragoza, España (2008)

Nickol, C.; Guynn, M.; Kohout, L. y Ozoroski, T. High Altitude Long Endurance Air Vehicle Analysis of Alternatives and Technology Requirements Development. 45 th Aerospace Sciences Meeting and Exhibit, Reno, Estados Unidos, 8 al11 de enero (2007)

Palethorpe, B.; Catucci, M.; Ede, J. y Jewell, G.W. Very Long Endurance Propulsion Systems. 3ª SEAS DTC Technical Conference, Edinburgh, UK (2008)

Payri, F. y Desantes, J.M. Motores de Combustión Interna Alternativos. 1a edición, Reverte, Barcelona, España (2011)

Raymer, D.P. Aircraft Design: A Conceptual Approach. 4aㅡ edición, AIAA Education, Estados Unidos (2006)

Sala, J.M. y López, L.M. Termodinámica fundamental. 3aㅗ edición, publicaciones Universidad de la Rioja, España (2000)

Scholz, D. The Standard Handbook for Aeronautical and Astronautical Engineers. 1ª edición, McGraw-Hill, New York, Estados Unidos (2003)

Torenbeck, E. Synthesis of Subsonic Airplane Design, $1^{\underline{a}}$ edición, Delft University Press, Delft, Holland (1982)

Tsonis, A. An Introduction to Atmospheric Thermodynamics. 2ª edición, Cambridge University Press, New York, Estados Unidos (2007)

Tulapurkara, E.G. Flight Dynamics-I (Performance Analysis). 1aㅡ edición, Dept. of Aerospace Engg. Indian Institute of Technology, Madras, India (2008)

Wallace, J.; Hobbs, P., Atmospheric Science. An Introductory Survey. Academic Press. (1997) 
